This usually applies to indications for a particular procedure and probably stems from the textbook having such a large authorship. Nevertheless, this certainly does not detract from the overall quality of the text and can lead to useful discussion points with colleagues.

In summary, this book can confidently be recommended to all students, practitioners and specialists in training. It fully achieves the goal of providing a contemporary, comprehensive and very readable account of paediatric clinical practice.

A. Gow

\section{PEDIATRIC DENTISTRY: INFANCY THROUGH ADOLESCENCE, 5TH EDITION}

P. S. Casamassimo,
H. W. Fields. D. J. McTigue,
A. J. Nowak
Elsevier
price £75.99; pp 648
ISBN 9780323085465

This is the fifth publication of an up to date textbook on paediatric dentistry. By virtue of its preeminent contributors, a broad range of subjects are covered. It is a comprehensive version, consisting of 648 pages with 520 high quality colour photographs and many informative tables, all of which are easy to consider and understand. The aim of the book is to become a discerning reference for paediatric dentistry. Initially, the book is comprised of 11 chapters on fundamental paediatric dentistry. It is then organised in a unique age specific criteria discussing all aspects of paediatric dentistry from infancy through to adolescence. Each age specific section covers the physical, cognitive, emotional and social changes that children experience, along with the epidemiology of dental disease at that age.

Overall, the book is comprised of five parts with 40 chapters discussing the examination, treatment planning, radiographic concerns, prevention, trauma, restorative dentistry, pulp therapy, orthodontics and behavioural management for each age range.

The publication endeavours to use behavioural management to highlight how to deliver appropriate dentistry to all age groups. This is particularly good for infants and age groups from 6-12 but a little less accomplished for adolescents. This will encourage practitioners to refresh their knowledge and technique.

Despite the book's positive qualities there are some notable problems because the book relates to American criteria and guidelines. References are from mainly American or Israeli journals. Significantly, at risk children receives only one minor paragraph with no help or advice for the British practitioner. Medical emergencies and sedation use American guidelines and the issue of consent refers to 'different state laws' with no reference to Gillick competency. Interestingly, there is virtually no mention of acid erosion (its aetiology or treatment), highlighting the divide which exists between ourselves and America. Additionally, although this book is comprehensive in some areas, such as the section on soft tissue lesions, it does lack depth in others, for instance, the treatment of ectopic canines. Overall, this is an accomplished reference book that can be used to improve your knowledge on this subject. It covers all crucial aspects of paediatric dentistry and I am certain that there will be something for everybody in this book.

F. Fletcher

\section{THE INCOMPARABLE CARIES OR A BOOK ABOUT TOOTH DECAY BY AN EXTRAORDINARY ARTIST!}

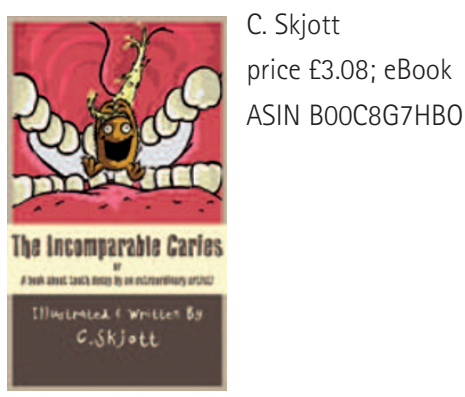

A novel account of the caries process from the perspective of none other than 'Caries' himself! The story depicts a young boy named Ollie who through frequent sugar consumption and a lackadaisical approach to oral hygiene finds himself the canvas for the artist Caries and his aptly named friends; Lac, St', and Nei to create their masterpieces - tooth cavities.
With the ever-increasing sugar content of the modern diet it is particularly important to educate both children and parents of the associated risk of developing tooth decay. This beautifully illustrated e-book really captures the imagination and delivers these key messages of healthy eating and excellent oral hygiene in both a humorous and understandable way. We follow Ollie through a typical day involving family meal times, schoolwork and recreation with friends, providing several situations for children to relate to. The author directly involves the reader by posing the question 'Have you ever felt sticky stuff on your teeth when you haven't brushed your teeth?' reinforcing that caries affects everyone.

The story concludes with an emergency trip to the dentist as Ollie has toothache. Ollie is frightened initially but the dentist is kind and the treatment is explained and successfully performed providing a positive experience, which would be a beneficial modeling tool in behaviour management.

Children will find the delightfully disgusting illustrations a visual treat whilst receiving both reinforcement of the aetiological factors in the caries process and some key preventive oral hygiene advice to stop Caries and his friends making masterpieces out of their own teeth.

I believe this book would appeal to a variety of children, the bright illustrations appealing to younger children and the inclusion of simple tooth anatomy and more complex language to a slightly older age group; although some concepts such as the bacterial origins of Caries' friends' names may not be understood. I feel this book would make a valuable teaching aid for the delivery of oral hygiene in a classroom setting or as an addition to any reading list, and whilst it may not fully comply with the Department of Health guidelines and would benefit from some rewording for the British audience, it still makes for an interesting read.

I shall leave you with Caries' chilling message to take care of your teeth or he will!

R. Parry 